\title{
Determination of Oxidized Phosphatidylcholines by Hydrophilic Interaction Liquid Chromatography Coupled to Fourier Transform Mass Spectrometry
}

Pia Sala ${ }^{1,2, \dagger}$, Sandra Pötz ${ }^{1,2, \dagger}$, Martina Brunner ${ }^{1}$, Martin Trötzmüller ${ }^{1}$, Alexander Fauland ${ }^{1,2}$, Alexander Triebl ${ }^{1}$, Jürgen Hartler ${ }^{3,4}$, Ernst Lankmayr ${ }^{2}$ and Harald C. Köfeler ${ }^{1,4, *}$

1 Core Facility for Mass Spectrometry, Medical University of Graz, Stiftingtalstrasse 24, Graz 8010, Austria; E-Mails: pia.sala@tu-dresden.de (P.S.); sandra.poetz@pccl.at (S.P.); martina.brunner@medunigraz.at (M.B.); martin.troetzmueller@medunigraz.at (M.T.); alexander.fauland@ki.se (A.F.); alexander.triebl@medunigraz.at (A.T.)

2 Institute of Analytical Chemistry and Food Chemistry, Graz University of Technology, Stremayrgasse 9/II, Graz 8010, Austria; E-Mail: lankmayr@tugraz.at

3 Bioinformatics Group, Institute for Knowledge Discovery, Graz University of Technology, Petersgasse 14, Graz 8010, Austria; E-Mail: juergen.hartler@tugraz.at

4 Omics Center Graz, Stiftingtalstrasse 24, Graz 8010, Austria

$\dagger$ These authors contributed equally to this work.

* Author to whom correspondence should be addressed; E-Mail: harald.koefeler@medunigraz.at; Tel.: +43-316-385 (ext. 73005); Fax: +43-316-385 (ext. 73009).

Academic Editor: Laszlo Prokai

Received: 5 March 2015 / Accepted: 8 April 2015 / Published: 14 April 2015

\begin{abstract}
A novel liquid chromatography-mass spectrometry (LC-MS) approach for analysis of oxidized phosphatidylcholines by an Orbitrap Fourier Transform mass spectrometer in positive electrospray ionization (ESI) coupled to hydrophilic interaction liquid chromatography (HILIC) was developed. This method depends on three selectivity criteria for separation and identification: retention time, exact mass at a resolution of 100,000 and collision induced dissociation (CID) fragment spectra in a linear ion trap. The process of chromatography development showed the best separation properties with a silica-based Kinetex column. This type of chromatography was able to separate all major lipid classes expected in mammalian samples, yielding increased sensitivity of oxidized phosphatidylcholines over reversed phase chromatography. Identification of molecular species was achieved by exact mass on intact molecular ions and CID tandem mass spectra containing characteristic fragments.
\end{abstract}


Due to a lack of commercially available standards, method development was performed with copper induced oxidation products of palmitoyl-arachidonoyl-phosphatidylcholine, which resulted in a plethora of lipid species oxidized at the arachidonoyl moiety. Validation of the method was done with copper oxidized human low-density lipoprotein (LDL) prepared by ultracentrifugation. In these LDL samples we could identify 46 oxidized molecular phosphatidylcholine species out of 99 possible candidates.

Keywords: fourier transform orbitrap mass spectrometry; hydrophilic interaction liquid chromatography; oxidized low density lipoprotein; oxidized phospholipids; LC-MS

\section{Introduction}

Non-enzymatic cellular oxidation processes are triggered by reactive oxygen species (ROS) such as hydrogen peroxide, superoxide anions or peroxide anions produced in the respiratory chain in mitochondria. Under normal cellular conditions ROS are immediately eliminated by various antioxidants, but in case of an imbalance in the antioxidant system excessive ROS may react with a wide variety of biomolecules and pave the way to certain pathophysiological conditions [1]. One compound class highly susceptible to ROS are polyunsaturated fatty acids (PUFA), particularly when esterified to membrane phospholipids in close proximity to ROS production. This lipid peroxidation process preferably starts at bis-allylic methylene groups in PUFA and leads to a plethora of molecular phospholipid oxidation products, containing hydroxy, hydroperoxy, ketene, aldehyde and carboxylic acid groups $[2,3]$. Furthermore, truncation of the esterified PUFA at various positions adds another layer of molecular complexity. Due to their wide variety of chemical functionalities, oxidized phospholipids do not cause a singular effect in cellular systems but act in a plethora of mechanisms, sometimes even contradictory in the physiological sense [4]. Therefore, oxidized phospholipids are involved in many different pathophysiological processes, resulting in diseases like atherosclerosis, acute inflammation, ischemia and multiple sclerosis, to name just a few $[5,6]$.

Since only minute amounts of oxidized lipid species are to be expected in biological systems, analysis of oxidized phospholipids is a challenging task. Due to its high sensitivity and selectivity, mass spectrometry coupled to liquid chromatography is a suitable technology for this challenge [7]. At the chromatography end, most groups work with reversed phase HPLC relying on separation by fatty acyl hydrophobicity [8-10], while only a few use normal phase separation $[11,12]$ or a combination of both polarities in a 2D approach [13]. The advantage of recently emerging hydrophilic interaction liquid chromatography (HILIC) separation techniques in lipid analysis is the ability to separate phospholipids by their polar head groups rather than by their non-polar fatty acyl moieties [14-16], which is important for lipids with multiple functional groups like oxidized phospholipids. Another substantial advantage is "pseudo normal phase separation" using aqueous solvent mixtures compatible with the electrospray ionization (ESI) process. At the mass spectrometry end, most conventional platforms use low resolution instrumentation (ion trap, triple quadrupole) relying on retention time and characteristic fragments [8-10,13]. Nevertheless, the analytical power for determination of oxidized phospholipids can be tremendously increased by the use of high resolution equipment $[17,18]$, even 
more so by Fourier transform-mass spectrometry (FT-MS) instruments based either on ion cyclotron resonance or on Orbitrap technology [19-22].

We matched the challenges of oxidized phospholipid analysis by development of a liquid chromatography-mass spectrometry (LC-MS) method based on HILIC separation, high resolution Orbitrap mass spectrometry on intact molecular ions and by automated data dependent linear ion trap fragment spectra. The instrumental setting is complemented by data processing with a Lipid Data Analyzer, a customized in-house developed software solution [23].

\section{Results and Discussion}

\subsection{HPLC and Mass Spectrometry Development}

One major aim of this study was the development of a method for separation of oxidized phosphatidylcholines from non-oxidized, as well as from other, lipid classes. The challenge of this endeavor was on the one hand, the similar polarity of some oxidized and native phospholipids, and on the other hand the big range of oxidized species polarity to be covered. We focused just on phosphatidylcholine, since it is the most abundant phospholipid class in biological membranes and consequently most oxidized phospholipids are to be expected from this lipid class. For the sake of simplicity we used only oxidized 1-palmitoyl-2-arachidonyl-phosphatidylcholine (PAPC; PC 16:0/20:4) for chromatographic method development. Oxidation of PAPC results in an ample array of oxidized products ranging from truncated species with only five carbon atoms at $s n-2$ position to complex rearrangements of arachidonic acid to isoprostaglandins, isolevuglandins and isothromboxanes [2]. This artificial mix fulfilled our demands for a wide range of polarities and functional groups well, and we could identify 36 oxidized species originating from PAPC (Supplementary Table S1). Additionally to these oxidized PAPC species, we used a set of defined lipid standards (Supplementary Table S2) for development of chromatography. We started chromatographic development with an YMC Diol HILIC column but finally switched to a Kinetex HILIC column since this column resulted in better chromatographic resolution. Due to the huge range of oxidized $s n-2$ fatty acyl chain lengths and functional groups it was not possible to elute all oxidized PAPC species in just one defined chromatographic peak as we initially aimed for, but nevertheless oxidized species are sufficiently separated from the non-oxidized species (Figure 1) to avoid the adverse effects of ion suppression of co-eluting compounds as is the case in all shotgun methods [20-22]. Furthermore we were able to add another layer of separation by high resolution mass spectrometry $(\mathrm{R}=100,000$ at $\mathrm{m} / \mathrm{z} 400)$, for detecting lipid species which are unresolved by chromatography, thereby resulting in confident information about elemental compositions by exact mass on molecular ions. Figure 2 exemplifies the advantages of our high resolution mass spectrometry platform by detection of a minor oxidized phospholipid compound (PC 18:0_10:3[2O]) at the same nominal mass and retention time as a major lipid $(\mathrm{M}+1$ peak of $N$-palmitoyl-sphingomyelin). The maximum mass resolution needed for separation of oxygenated $\left(\mathrm{C}_{\mathrm{n}-1} \mathrm{H}_{\mathrm{m}-4} \mathrm{O} 9 \mathrm{NP}\right)$ from non-oxygenated $\left(\mathrm{C}_{\mathrm{n}} \mathrm{H}_{m} \mathrm{O}_{8} \mathrm{NP}\right)$ phosphatidylcholine (PC) or phosphatidylethanolamine (PE) compounds would be at least 22,000 at $\mathrm{m} / \mathrm{z} 800$, which is easily achieved by Orbitrap mass spectrometry. This is clearly an advantage over low-resolution instrumentation, and provides a high 
degree of certainty even without collision induced activation (CID) fragmentation such as used in low-resolution single reaction monitoring (SRM) methods $[8,10,20]$.

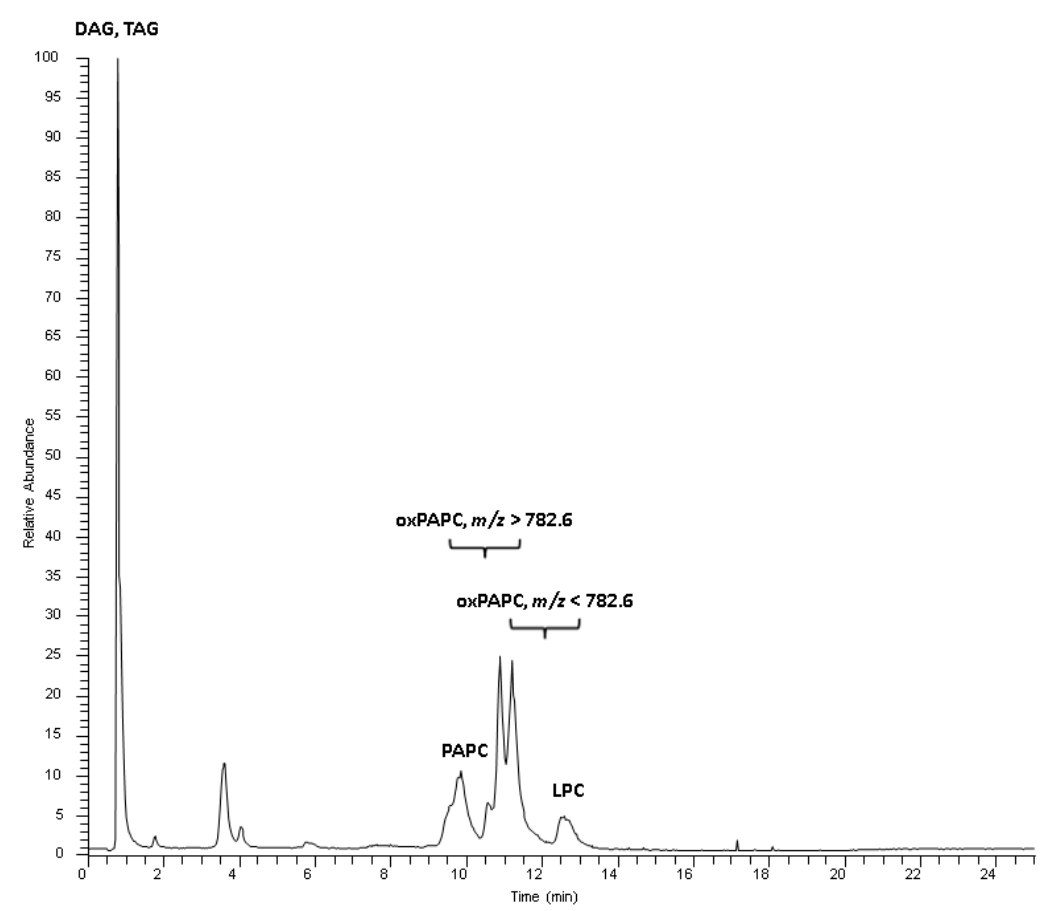

Figure 1. Total ion chromatogram of a standard mix containing oxidized and non-oxidized PAPC. Oxidized PAPC species elute according to polarity and carbon number of the oxidized arachidonic acid moiety between the non-oxidized PAPC and lysophosphatidylcholines (LPC). Triacylglycerols (TAG) and diacylglycerols (DAG) elute with the void volume.

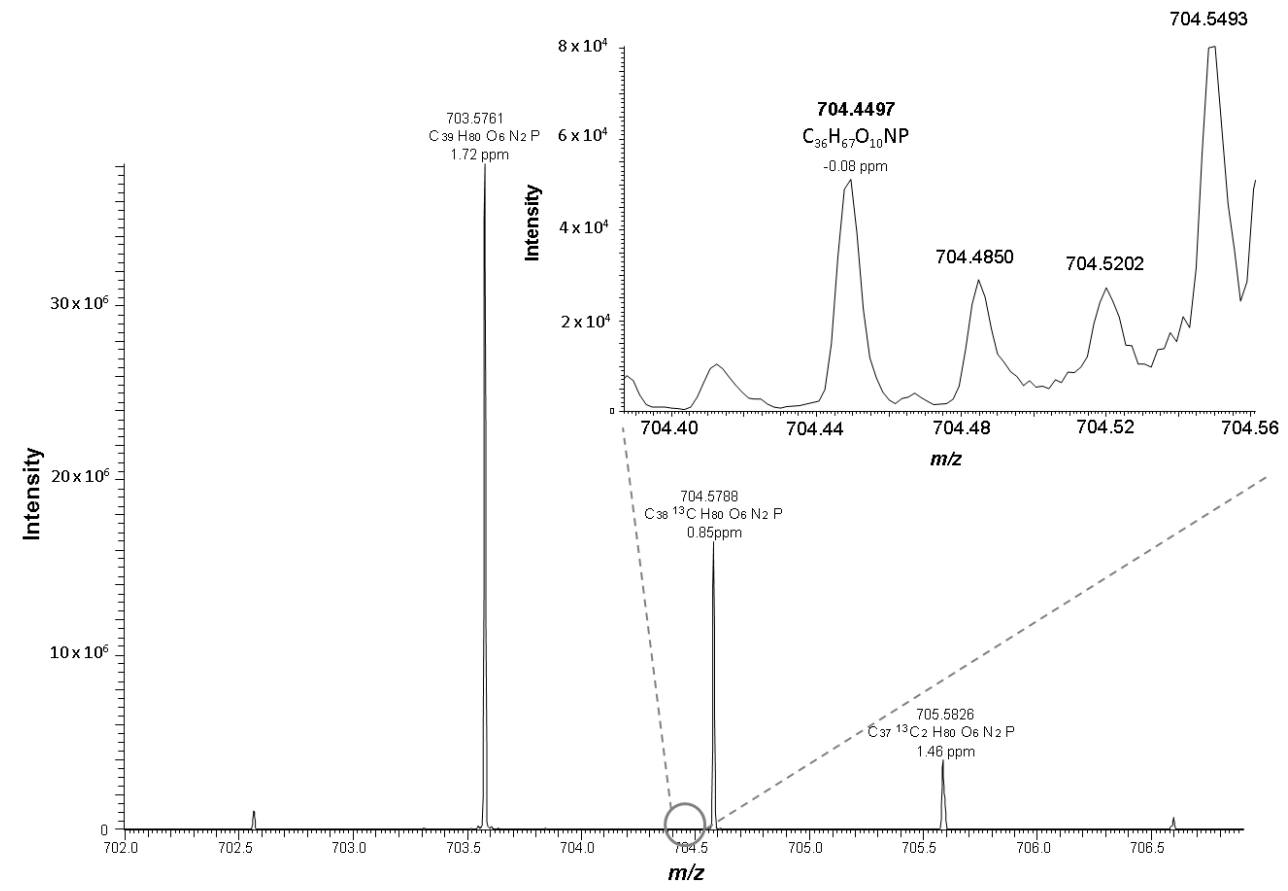

Figure 2. Positive ESI mass spectrum of oxidized LDL at the retention time $11.38 \mathrm{~min}$. In the depicted spectrum PC 18:0_10:3[2O] at $m / z 704.4497$ co-elutes at the same retention time as SM 16:0 and has the same nominal mass as the M+1 peak of SM 16:0 $(\mathrm{m} / \mathrm{z} 704.5788)$. 


\subsection{Identification Strategy for Oxidized Phospholipids}

Unambiguous structural identification of lipids in general and oxidized phospholipids in particular is hardly achieved with conventional LC-MS or matrix assisted laser desorption ionization-time of flight (MALDI-TOF) equipment, because low energy CID or post source decay (PSD) spectra rarely cover in depth structural features like stereochemistry. For a deeper coverage of structural details like double bond position or formation of ring structures, one would need high energy CID (four sector MS, MALDI-TOF/TOF) or electron impact ionization, both enabling charge remote fragmentations [24,25], or ozonolysis for location of double bonds [26]. Additionally, nuclear magnetic resonance spectroscopy could support in depth stereochemical structural elucidation [12]. The trade-off of the aforementioned methods is often a compromised sensitivity and/or missing chromatography.

Bearing this in mind we identified oxidized phosphatidylcholines as comprehensively as possible at uncompromised sensitivity and applicability of the method in terms of acquisition speed. The aim of establishing the heuristic scoring system depicted in Table 1 is to provide an estimation of identification certainty at a glance. The scoring system relies on exact mass of the molecular precursor and specific fragments in low resolution linear ion trap CID spectra. In a first step, we evaluated whether the elemental composition displayed in each extracted 3D ion chromatogram would fit with any oxidized phosphatidylcholine species. To this end, we generated a mass list of all literature of known oxidized phosphatidylcholine species and some additional likely elemental compositions and identified all matching mass peaks at a mass tolerance of 5 ppm by Lipid Data Analyzer software. Out of these candidates, only the ones with less than 2 ppm mass deviation gained a scoring point. In a second step, we took MS/MS spectra from data dependent analysis into account. If the top 10 methods did not either trigger MS/MS on a precursor, or only (mixed) MS/MS spectra of too low quality were available, the candidate was rejected and resulted in just one scoring point. If MS/MS spectra of sufficient quality were available, a scoring point was added for head group specific fragments indicative for PC. These fragments were either the choline phosphate head group at $\mathrm{m} / \mathrm{z} 184$ or the neutral loss of $59 \mathrm{Da}$. Therefore identification as oxidized PC species according to the described scoring system was achieved if at least two scoring points were assigned. The next two scoring points were assigned if the neutral losses of the presumably oxidized fatty acid moiety were detected, one for the fatty acyl $\left(\mathrm{R}_{\mathrm{ox}}-\mathrm{COOH}\right)$ loss and another one for the corresponding ketene $\left(\mathrm{R}_{\mathrm{ox}}-\mathrm{C}=\mathrm{O}\right)$ loss. At this stage, it was possible to assign fatty acids in the molecular structure. Additionally 0.5 scoring points were assigned for a neutral loss of one or two $\mathrm{H}_{2} \mathrm{O}$. This neutral loss is considered to be non-specific, which is reflected by only half the scoring weight of 0.5 instead of 1.0 points in the scoring system. Nevertheless, unmodified PC compounds do not show any $\mathrm{H}_{2} \mathrm{O}$ loss and therefore these fragments could indicate additional hydroxy groups or rearranged carbonyl functionalities, which corroborates the identity of an oxidized PC. We did not take into account retention times in our scoring system because chemically pure standard compounds would have been required for each oxidized PC species. Since only a few oxidized PC species are commercially available, this was not an option. Despite these constraints, we ensured that retention time and proposed structure would approximately fit together in terms of polarity. 
Table 1. Presented scoring scheme to estimate the reliability of a hit.

\begin{tabular}{ccc}
\hline Requirement & Fragment & Scoring Points \\
\hline Exact mass $<2 \mathrm{ppm}$ & & 1.0 \\
Headgroup fragment & {$[\mathrm{PChol}]^{+}$or $[\mathrm{M}-59]^{+}$} & 1.0 \\
Fatty acyl fragment & {$\left[\mathrm{M}-\mathrm{R}_{\mathrm{ox}}\right]^{+}$} & 1.0 \\
Fatty acyl fragment & {$\left[\mathrm{M}-\mathrm{R}_{\mathrm{ox}}=\mathrm{C}=\mathrm{O}\right]^{+}$} & 1.0 \\
Water loss at oxidized moiety & {$\left[\mathrm{M}-\mathrm{H}_{2} \mathrm{O}\right]^{+}$} & 0.5 \\
Water loss at oxidized moiety & {$\left[\mathrm{M}-2 \mathrm{H}_{2} \mathrm{O}\right]^{+}$} & 0.5 \\
\hline
\end{tabular}

\subsection{Analysis of Oxidized Phosphatidylcholine Species in Oxidized Low Density Lipoprotein}

Finally, we evaluated our method with $\mathrm{Cu}^{2+}$ oxidized low-density lipoprotein (LDL). The Lipid Data Analyzer (LDA) identified 99 possible oxidized PC candidates by exact mass and isotopic pattern in 3D mass chromatograms ( $\mathrm{m} / \mathrm{z}$, retention time, and intensity). Even though LDA would have supported retention time constraints [23], they were not set in this analysis since the retention time of the compounds is not exactly known. Out of these candidates, 46 compounds could be identified unambiguously as oxidized PC compounds, and 28 of them could be assigned with fatty acid composition (Table 2). Finally, we matched the species in the higher scoring ranks with known possible structures from the literature to assign potential candidate structures for the detected lipid species annotated in shorthand nomenclature (Table 3) [27]. In this sense, our platform provides a good starting point for further unambiguous structural in-depth confirmation of the identified oxidized PC species. In comparison to our existing reversed phase method [28], the HILIC method delivered a deeper coverage of oxidized PCs (46 vs. 22 species). This might be due to better chromatographic separation and subsequently less ionization suppression effects. These results compare well to existing results [8,9], but certainty is boosted by exact mass on molecular ions. The relative contributions to the total pool of oxidized PC (Table 2) revealed linoleic acid containing PC species to be the main precursors of oxidized PC. Furthermore the majority (73.8\%) of oxidized PC species were non-truncated oxygenated compounds. This indicates a rather mild oxidation process including some rearrangements in the PUFA, but rather few $\mathrm{C}-\mathrm{C}$ bond cleavages.

Table 2. List of observed exact masses, elemental compositions, retention times (RT) and corresponding compound assignments by shorthand nomenclature in oxidized LDL. Relative contribution is calculated by peak areas of mass chromatograms where the sum of all oxidized PC species detected equals $100 \%$. Score is calculated according to the scheme in Table 1.

\begin{tabular}{ccccccc}
\hline Species & $\begin{array}{c}\text { Elemental } \\
\text { Composition }\end{array}$ & $\begin{array}{c}\boldsymbol{m} / \boldsymbol{z} \text { Observed } \\
{[\mathbf{M}+\mathbf{H}]^{+}}\end{array}$ & $\boldsymbol{\Delta} \mathbf{p p m}$ & $\begin{array}{c}\text { RT } \\
{[\mathbf{m i n}]}\end{array}$ & $\begin{array}{c}\text { Relative } \\
\text { Contribution [\%] }\end{array}$ & Score \\
\hline PC 16:0_20:4 [4O] & $\mathrm{C}_{44} \mathrm{H}_{80} \mathrm{O}_{12} \mathrm{NP}$ & 846.5502 & 1.30 & 10.23 & 0.91 & 5.0 \\
PC 16:0_20:3 [4O] & $\mathrm{C}_{44} \mathrm{H}_{82} \mathrm{O}_{12} \mathrm{NP}$ & 848.5655 & 0.93 & 10.38 & 0.71 & 5.0 \\
PC 18:0_20:5 [2O] & $\mathrm{C}_{46} \mathrm{H}_{82} \mathrm{O}_{10} \mathrm{NP}$ & 840.5750 & 0.15 & 10.09 & 0.54 & 5.0 \\
PC 18:0_20:4[3O] & $\mathrm{C}_{46} \mathrm{H}_{84} \mathrm{O}_{11} \mathrm{NP}$ & 858.5850 & 0.57 & 9.64 & 3.40 & 5.0 \\
PC 18:0_20:4[4O] & $\mathrm{C}_{46} \mathrm{H}_{84} \mathrm{O}_{12} \mathrm{NP}$ & 874.5793 & -1.11 & 10.06 & 0.83 & 5.0 \\
PC 16:0_5:1 [O] & $\mathrm{C}_{29} \mathrm{H}_{56} \mathrm{O}_{9} \mathrm{NP}$ & 594.3770 & 0.82 & 11.82 & 1.48 & 4.5 \\
\hline
\end{tabular}


Table 2. Cont.

\begin{tabular}{|c|c|c|c|c|c|c|}
\hline Species & $\begin{array}{c}\text { Elemental } \\
\text { Composition }\end{array}$ & $\begin{array}{c}m / z \text { Observed } \\
{[\mathrm{M}+\mathrm{H}]^{+}} \\
\end{array}$ & $\Delta$ ppm & $\begin{array}{c}\text { RT } \\
{[\mathrm{min}]}\end{array}$ & $\begin{array}{c}\text { Relative } \\
\text { Contribution [\%] } \\
\end{array}$ & Score \\
\hline PC 16:0_8:3 [3O] & $\mathrm{C}_{32} \mathrm{H}_{58} \mathrm{O}_{11} \mathrm{NP}$ & 664.3829 & 1.33 & 12.20 & 0.01 & 4.5 \\
\hline PC 16:0_8:2 [3O] & $\mathrm{C}_{32} \mathrm{H}_{60} \mathrm{O}_{11} \mathrm{NP}$ & 666.3986 & 1.33 & 11.48 & 0.03 & 4.5 \\
\hline PC 16:0_22:4 [O] & $\mathrm{C}_{46} \mathrm{H}_{84} \mathrm{O}_{9} \mathrm{NP}$ & 826.5960 & -1.79 & 12.03 & 0.56 & 4.5 \\
\hline PC 16:0_4:1 [O] & $\mathrm{C}_{30} \mathrm{H}_{58} \mathrm{O}_{9} \mathrm{NP}$ & 608.3920 & 0.30 & 11.82 & 0.60 & 4.5 \\
\hline PC 16:0_6:1 [O] & $\mathrm{C}_{33} \mathrm{H}_{62} \mathrm{O}_{10} \mathrm{NP}$ & 664.4178 & 0.73 & 11.08 & 0.09 & 4.5 \\
\hline PC 16:0_9:2 [2O] & $\mathrm{C}_{46} \mathrm{H}_{84} \mathrm{O}_{9} \mathrm{NP}$ & 826.5960 & 0.52 & 9.65 & 0.16 & 4.5 \\
\hline PC 16:0_9:0 [O] & $\mathrm{C}_{33} \mathrm{H}_{64} \mathrm{O}_{9} \mathrm{NP}$ & 650.4402 & -1.69 & 10.86 & 7.38 & 4.0 \\
\hline PC 16:0_8:2 [2O] & $\mathrm{C}_{32} \mathrm{H}_{60} \mathrm{O}_{10} \mathrm{NP}$ & 650.4036 & 1.43 & 11.22 & 0.13 & 4.0 \\
\hline PC 16:0_20:6 [2O] & $\mathrm{C}_{44} \mathrm{H}_{76} \mathrm{O}_{10} \mathrm{NP}$ & 810.5290 & 1.24 & 10.23 & 1.71 & 4.0 \\
\hline PC 16:0_20:5 [3O] & $\mathrm{C}_{44} \mathrm{H}_{78} \mathrm{O}_{11} \mathrm{NP}$ & 828.5393 & 0.91 & 10.06 & 3.28 & 4.0 \\
\hline PC 16:0_20:4 [3O] & $\mathrm{C}_{44} \mathrm{H}_{80} \mathrm{O}_{11} \mathrm{NP}$ & 830.5552 & 1.27 & 9.81 & 6.08 & 4.0 \\
\hline PC 16:0_20:3 [3O] & $\mathrm{C}_{44} \mathrm{H}_{82} \mathrm{O}_{11} \mathrm{NP}$ & 832.5687 & -1.25 & 10.01 & 3.02 & 4.0 \\
\hline PC 16:0_22:6 [2O] & $\mathrm{C}_{46} \mathrm{H}_{80} \mathrm{O}_{10} \mathrm{NP}$ & 838.5590 & 0.29 & 10.06 & 1.41 & 4.0 \\
\hline PC $16: 0 \_12: 2[2 \mathrm{O}]$ & $\mathrm{C}_{36} \mathrm{H}_{68} \mathrm{O}_{10} \mathrm{NP}$ & 706.4650 & 0.52 & 10.97 & 0.06 & 3.5 \\
\hline PC 16:0_18:2 [2O] & $\mathrm{C}_{42} \mathrm{H}_{80} \mathrm{O}_{10} \mathrm{NP}$ & 790.5577 & 1.85 & 10.04 & 15.86 & 3.5 \\
\hline PC 18:0_9:0 [O] & $\mathrm{C}_{35} \mathrm{H}_{68} \mathrm{O}_{9} \mathrm{NP}$ & 678.4714 & -1.53 & 10.68 & 5.08 & 3.5 \\
\hline PC 18:0_18:2 [O] & $\mathrm{C}_{44} \mathrm{H}_{84} \mathrm{O}_{9} \mathrm{NP}$ & 802.5946 & 1.22 & 9.81 & 6.12 & 3.5 \\
\hline PC 16:0_20:4 [O] & $\mathrm{C}_{44} \mathrm{H}_{80} \mathrm{O}_{9} \mathrm{NP}$ & 798.5651 & 1.03 & 9.81 & 0.94 & 3.5 \\
\hline PC 18:0_5:1 [O] & $\mathrm{C}_{31} \mathrm{H}_{60} \mathrm{O}_{9} \mathrm{NP}$ & 622.4080 & -0.29 & 11.62 & 1.90 & 3.5 \\
\hline PC 18:0_5:1 [2O] & $\mathrm{C}_{31} \mathrm{H}_{60} \mathrm{O}_{10} \mathrm{NP}$ & 638.4027 & 0.00 & 10.74 & 0.20 & 3.5 \\
\hline PC 16:0_10:3 [2O] & $\mathrm{C}_{34} \mathrm{H}_{62} \mathrm{O}_{10} \mathrm{NP}$ & 676.4193 & -1.44 & 11.10 & 0.08 & 3.5 \\
\hline PC 18:0_10:3 [2O] & $\mathrm{C}_{36} \mathrm{H}_{66} \mathrm{O}_{10} \mathrm{NP}$ & 704.4497 & -0.08 & 10.49 & 0.18 & 3.5 \\
\hline PC 36:5 [2O] & $\mathrm{C}_{44} \mathrm{H}_{78} \mathrm{O}_{10} \mathrm{NP}$ & 812.5440 & -0.53 & 10.23 & 1.57 & 3.0 \\
\hline PC $38: 6$ [2O] & $\mathrm{C}_{46} \mathrm{H}_{80} \mathrm{O}_{10} \mathrm{NP}$ & 838.5590 & -0.29 & 10.10 & 1.61 & 3.0 \\
\hline $\mathrm{PC} 40: 6[\mathrm{O}]$ & $\mathrm{C}_{48} \mathrm{H}_{84} \mathrm{O}_{9} \mathrm{NP}$ & 850.5960 & -0.50 & 10.70 & 0.10 & 2.5 \\
\hline PC 34:2 [O] & $\mathrm{C}_{42} \mathrm{H}_{80} \mathrm{O}_{9} \mathrm{NP}$ & 774.5643 & -0.08 & 9.98 & 20.03 & 2.5 \\
\hline PC 27:0 [2O] & $\mathrm{C}_{35} \mathrm{H}_{68} \mathrm{O}_{10} \mathrm{NP}$ & 694.4650 & 0.53 & 10.87 & 1.30 & 2.5 \\
\hline PC $36: 4[2 \mathrm{O}]$ & $\mathrm{C}_{44} \mathrm{H}_{80} \mathrm{O}_{10} \mathrm{NP}$ & 814.5590 & 0.30 & 9.75 & 5.02 & 2.5 \\
\hline PC 26:3 [2O] & $\mathrm{C}_{34} \mathrm{H}_{62} \mathrm{O}_{10} \mathrm{NP}$ & 676.4183 & 0.00 & 10.86 & 0.08 & 2.5 \\
\hline PC 26:3 [3O] & $\mathrm{C}_{34} \mathrm{H}_{62} \mathrm{O}_{11} \mathrm{NP}$ & 692.4130 & 0.44 & 12.75 & 0.03 & 2.5 \\
\hline PC 26:2 [3O] & $\mathrm{C}_{34} \mathrm{H}_{64} \mathrm{O}_{11} \mathrm{NP}$ & 694.4284 & 0.70 & 10.94 & 0.06 & 2.5 \\
\hline PC 20:1 [2O] & $\mathrm{C}_{28} \mathrm{H}_{54} \mathrm{O}_{10} \mathrm{NP}$ & 596.3548 & 1.64 & 11.73 & 0.03 & 2.5 \\
\hline PC 22:1 [2O] & $\mathrm{C}_{30} \mathrm{H}_{58} \mathrm{O}_{10} \mathrm{NP}$ & 624.3871 & 0.00 & 11.56 & 0.09 & 2.5 \\
\hline PC 27:3 [O] & $\mathrm{C}_{35} \mathrm{H}_{64} \mathrm{O}_{9} \mathrm{NP}$ & 674.4381 & 1.45 & 11.75 & 0.07 & 2.5 \\
\hline PC 36:6 [O] & $\mathrm{C}_{44} \mathrm{H}_{76} \mathrm{O}_{9} \mathrm{NP}$ & 794.5325 & 0.62 & 11.24 & 0.11 & 2.5 \\
\hline PC 25:0 [2O] & $\mathrm{C}_{33} \mathrm{H}_{64} \mathrm{O}_{10} \mathrm{NP}$ & 666.4351 & -1.56 & 10.55 & 1.31 & 2.0 \\
\hline PC 28:3 [3O] & $\mathrm{C}_{36} \mathrm{H}_{66} \mathrm{O}_{11} \mathrm{NP}$ & 720.4446 & 0.00 & 10.38 & 0.06 & 2.0 \\
\hline PC 21:1 [2O] & $\mathrm{C}_{29} \mathrm{H}_{56} \mathrm{O}_{10} \mathrm{NP}$ & 610.3720 & 0.91 & 12.43 & 0.11 & 2.0 \\
\hline PC 34:6 [O] & $\mathrm{C}_{42} \mathrm{H}_{72} \mathrm{O}_{9} \mathrm{NP}$ & 766.5020 & -0.40 & 12.74 & 0.03 & 2.0 \\
\hline $\mathrm{PC} 24: 2[\mathrm{O}]$ & $\mathrm{C}_{32} \mathrm{H}_{60} \mathrm{O}_{9} \mathrm{NP}$ & 634.4088 & -1.64 & 11.45 & 0.06 & 2.0 \\
\hline
\end{tabular}


Table 3. List of detected fragments in oxidized LDL including assignment of possible non-oxidized precursors and oxidized PC structures reported in the literature for the corresponding shorthand denoted compounds. Abbreviations of oxidized structures are explained in Supplementary Table S3.

\begin{tabular}{|c|c|c|c|c|c|c|}
\hline Species & $\begin{array}{c}\text { Possible Oxidized } \\
\text { Structure } \\
\end{array}$ & $\begin{array}{l}\text { Possible } \\
\text { Precursor }\end{array}$ & $\begin{array}{c}{\text { [PChol }]^{+}}{ }_{[\text {M-59 }}^{+} \\
\end{array}$ & $\begin{array}{c}{\left[\mathbf{M}-\mathbf{R}_{\mathbf{o x}}\right]^{+}} \\
{\left[\mathbf{M}-\mathbf{R}_{\mathbf{o x}}=\mathbf{C}=\mathbf{O}\right]^{+}}\end{array}$ & $\begin{array}{r}{\left[\mathbf{M}-\mathbf{H}_{2} \mathbf{O}\right]^{+}} \\
{\left[\mathbf{M}-2 \mathbf{H}_{2} \mathbf{O}\right]^{+}}\end{array}$ & Score \\
\hline PC 16:0_20:4 [4O] & isoPGG2 & PC 16:0/20:4 & 787 & 496,478 & 828,810 & 5.0 \\
\hline PC 16:0_20:3 [4O] & isoTxA2 & PC 16:0/20:4 & 789 & 496,478 & 830,812 & 5.0 \\
\hline PC 18:0_20:5 [2O] & isoPGJ2/A2 & PC 18:0/20:4 & 781 & 524,506 & 822,804 & 5.0 \\
\hline PC 18:0_20:4 [3O] & $\begin{array}{c}\text { isoPGE }_{2} / \mathrm{D}_{2} \\
\text { isoLGE } 2 / \mathrm{D}_{2} \text { isoTXB }\end{array}$ & PC 18:0/20:4 & 799 & 524,506 & 840,822 & 5.0 \\
\hline PC 18:0_20:4 [4O] & isoPGG2 & PC 18:0/20:4 & 815 & 524,506 & 856,838 & 5.0 \\
\hline PC 16:0 5:1 [O] & OV & PC 16:0/20:4 & 184 & 496,478 & 576 & 4.5 \\
\hline PC 16:0 $8: 3[3 \mathrm{O}]$ & KOdiA & PC 16:0/20:4 & 184 & 496,478 & 646 & 4.5 \\
\hline PC 16:0 $8: 2$ [3O] & HOdiA & PC 16:0/20:4 & 184 & 496,478 & 648 & 4.5 \\
\hline PC 16:0_22:4 [O] & & PC 16:0/22:4 & 767 & 496,478 & 808 & 4.5 \\
\hline PC 16:0_4:1 [O] & & PC 16:0/22:6 & 184 & 496,478 & 562 & 4.5 \\
\hline PC 16:0_6:1 [O] & & PC 18:0/22:6 & 184 & 496,478 & 590 & 4.5 \\
\hline PC 16:0_9:2 [2O] & & PC 18:0/22:6 & 184 & 496,478 & 646 & 4.5 \\
\hline PC 16:0_9:0 [O] & $\mathrm{ON}$ & PC 16:0/18:2 & 184 & 496,478 & & 4.0 \\
\hline PC 16:0_8:2 [2O] & HOOA & PC 16:0/20:4 & 184 & 496,478 & & 4.0 \\
\hline PC 16:0_20:6 [2O] & $\mathrm{EC}$ & PC 16:0/20:4 & 184 & 496 & 792,774 & 4.0 \\
\hline PC 16:0_20:5 [3O] & EI & PC 16:0/20:4 & 769 & 496 & 810,792 & 4.0 \\
\hline PC 16:0_20:4 [3O] & $\begin{array}{c}\text { isoPGE }_{2} / \mathrm{D}_{2} \\
\text { isoLGE } 2 / \mathrm{D}_{2} \text { isoTXB }{ }_{2}\end{array}$ & PC 16:0/20:4 & 771 & 496 & 812,794 & 4.0 \\
\hline PC 16:0_20:3 [3O] & isoPGF $2 \alpha$ & PC 16:0/20:4 & 773 & 496 & 814,796 & 4.0 \\
\hline PC 16:0_22:6 [2O] & & PC 16:0/22:6 & 779 & 496 & 820,802 & 4.0 \\
\hline PC 16:0_12:2 [2O] & HODA & PC 16:0/18:2 & 184 & 478 & 688 & 3.5 \\
\hline PC 16:0_18:2 [2O] & HpODE & PC 16:0/18:2 & 184 & 496 & 772 & 3.5 \\
\hline PC 18:0_9:0 [O] & ON & PC 18:0/18:2 & 184 & 524 & 660 & 3.5 \\
\hline PC 18:0_18:2 [O] & HODE & PC 18:0/18:2 & 184 & 524 & 784 & 3.5 \\
\hline PC 16:0_20:4 [O] & HETE & PC 16:0/20:4 & 184 & 478 & 680 & 3.5 \\
\hline PC 18:0 5:1 [O] & OV & PC 18:0/20:4 & 184 & 524 & 604 & 3.5 \\
\hline PC $18: 0 \_5: 1[2 \mathrm{O}]$ & $\mathrm{G}$ & PC 18:0/20:4 & 184 & 506 & 620 & 3.5 \\
\hline PC 16:0_10:3 [2O] & & PC 16:0/22:6 & 184 & 496 & 658 & 3.5 \\
\hline PC 18:0_10:3 [2O] & & PC 18:0/22:6 & 184 & 506 & 686 & 3.5 \\
\hline PC $36: 5$ [2O] & & PC 16:0/20:4 & 753 & & 794,772 & 3.0 \\
\hline PC $38: 6$ [2O] & & PC 18:0/20:4 & 779 & & 820,802 & 3.0 \\
\hline PC 40:6 [O] & & PC 18:0/22:6 & 791 & & 832 & 2.5 \\
\hline PC 34:2 [O] & & PC 16:0/18:2 & 184 & & 756 & 2.5 \\
\hline PC 27:0 [2O] & & PC 18:0/18:2 & 184 & & 676 & 2.5 \\
\hline PC 36:4 [2O] & & PC 16:0/20:4 & 755 & & 796 & 2.5 \\
\hline PC $26: 3[2 \mathrm{O}]$ & & PC 18:0/20:4 & 184 & & 658 & 2.5 \\
\hline PC 26:3 [3O] & & PC 18:0/20:4 & 184 & & 674 & 2.5 \\
\hline PC 26:2 [3O] & & PC 18:0/20:4 & 184 & & 676 & 2.5 \\
\hline PC 20:1 [2O] & & PC 16:0/22:6 & 184 & & 578 & 2.5 \\
\hline
\end{tabular}


Table 3. Cont.

\begin{tabular}{|c|c|c|c|c|c|c|}
\hline Species & $\begin{array}{c}\text { Possible Oxidized } \\
\text { Structure }\end{array}$ & $\begin{array}{l}\text { Possible } \\
\text { Precursor }\end{array}$ & $\begin{array}{c}{\left[_{\text {PChol }]^{+}}\right.} \\
{\left[{\mathrm{M}-59]^{+}}^{+}\right.}\end{array}$ & $\begin{array}{c}{\left[\mathbf{M}-\mathbf{R}_{\mathbf{o x}}\right]^{+}} \\
{\left[\mathbf{M}-\mathbf{R}_{\mathbf{o x}}=\mathbf{C}=\mathbf{O}\right]^{+}}\end{array}$ & $\begin{array}{c}{\left[\mathbf{M}-\mathrm{H}_{2} \mathbf{O}\right]^{+}} \\
{\left[\mathbf{M}-2 \mathrm{H}_{2} \mathbf{O}\right]^{+}}\end{array}$ & Score \\
\hline PC 22:1 [2O] & & PC 18:0/22:6 & 184 & & 606 & 2.5 \\
\hline PC 27:3 [O] & & PC 18:0/22:6 & 184 & & 656 & 2.5 \\
\hline PC 36:6 [O] & & PC 18:0/22:6 & 184 & & 776 & 2.5 \\
\hline PC 25:0 [2O] & & PC 16:0/18:2 & 184 & & & 2.0 \\
\hline PC 28:3 [3O] & & PC 16:0/18:2 & 184 & & & 2.0 \\
\hline PC 21:1 [2O] & & PC 16:0/20:4 & 184 & & & 2.0 \\
\hline PC 34:6 [O] & & PC 16:0/22:6 & 184 & & & 2.0 \\
\hline PC 24:2 [O] & & PC 18:0/22:6 & 184 & & & 2.0 \\
\hline
\end{tabular}

In summary, we developed a HILIC-coupled high-resolution LTQ-Orbitrap method for the analysis of oxidized PC species including data-dependent MS/MS spectra. This method enabled us to identify 46 oxidized PC species by exact mass of molecular ions and selective fragments in oxidized LDL.

\section{Material and Methods}

\subsection{Materials}

All lipid standards were purchased from Larodan (Malmö, Sweden). A complete list is shown in Supplementary Table S1. Standard stock solutions were dissolved in chloroform/methanol 1:1 $(v / v)$ at a concentration of $1 \mathrm{mM}$ and stored at $-20{ }^{\circ} \mathrm{C}$. Lipid standard mixtures were prepared freshly every day in chloroform/methanol 1:1 $(v / v)$ at a concentration of $3 \mu \mathrm{M}$ and were used immediately.

Chloroform was HPLC grade, ammonium acetate and formic acid were analytical grade, all obtained from Merck KGaA (Darmstadt, Germany). 2-Propanol was LC-MS grade and supplied by Fluka (Steinheim, Germany). Methanol and acetonitrile (LC/MS grade), and 28\% ammonia p.a. were all purchased from Sigma-Aldrich Chemie GmbH (Steinheim, Germany). Nitrogen (purity 99.9990) was obtained from Air Liquide (Graz, Austria). Ultra pure water purified by a Milli-Q Gradient system (Millipore, Bedford, MA, USA) was used in all experiments (resistivity $>18 \mathrm{M} \Omega \mathrm{cm}$ ).

\subsection{Oxidation of PAPC}

PAPC was dissolved in $1 \mathrm{~mL}$ methanol with a concentration of $3.3 \mathrm{mg} / \mathrm{mL}$. The solution was evacuated in a SpeedVac (Thermo Fisher Scientific, San Jose, CA, USA), mixed with $1 \mathrm{~mL}$ of a $5 \mu \mathrm{M}$ $\mathrm{CuSO}_{4} \cdot 5 \mathrm{H}_{2} \mathrm{O}$-solution in PBS-buffer, vortexed for $5 \mathrm{~min}$ and was then sonicated in an ice/water bath for $30 \mathrm{~min}$. After incubation for $113 \mathrm{~h}$ at room temperature, the oxidation was quenched by addition of $2 \mathrm{~mL}$ chloroform/methanol $(2: 1)(v / v)$ and the resulting suspension was centrifuged at 1360 times $\mathrm{g}$ at room temperature for $5 \mathrm{~min}$. The upper aqueous phase was extracted two times with $1 \mathrm{~mL}$ chloroform/methanol (2:1) $(v / v)$ each. The lower organic phases were collected and washed two times with ultra pure water. The solvent of the organic phases was evaporated in a SpeedVac, the residue was re-dissolved in chloroform/methanol $(1: 1)(v / v)$, overlaid with nitrogen $(\mathrm{g})$ and stored at $-80{ }^{\circ} \mathrm{C}$ until use [29,30]. 


\subsection{Isolation and Oxidation of $L D L$}

LDL was isolated from the sera of healthy blood donors by ultracentrifugation in successive $\mathrm{KBr}$ gradients as described in detail [21] and stored at $4{ }^{\circ} \mathrm{C}$ until use. LDL (1.6 mL) was dialysed overnight in $600 \mathrm{~mL}$ phosphate buffered saline (PBS) in an ice/water bath before oxidation was performed. Following that, $250 \mu \mathrm{L}$ of dialysed LDL were mixed with $\mathrm{CuSO}_{4} \cdot 5 \mathrm{H}_{2} \mathrm{O}$ and PBS-puffer and the concentration of $\mathrm{CuSO}_{4}$ was adjusted to $40 \mu \mathrm{M}$. This mixture was incubated for $25 \mathrm{~h}$ at $37{ }^{\circ} \mathrm{C}$ in air for oxidation [30]. The reaction was quenched by the addition of $2 \mathrm{~mL}$ of chloroform/methanol $(2: 1)(v / v)$ and the lipids were extracted by a Folch extract [29]. After extraction the lipids were diluted in $200 \mu \mathrm{L}$ chloroform/methanol $(1: 1)(v / v)$ and stored at $-80{ }^{\circ} \mathrm{C}$ until use.

\subsection{High Performance Liquid Chromatography-Mass Spectrometry}

HILIC-HPLC was performed on an Accela HPLC system (Thermo Scientific, San Jose, CA, USA) equipped with a $100 \mathrm{~mm}$ length, $2.1 \mathrm{~mm}$ i.d. Kinetex column with $2.6 \mu \mathrm{m}$ particle size and $100 \AA$ pore size (Phenomenex, Aschaffenburg, Germany). Mobile phase A was water with $10 \mathrm{mM}$ ammonia acetate and $0.5 \%$ formic acid. Mobile phase B was acetonitrile/2-propanol 5:2 ( $v / v)$ containing $10 \mathrm{mM}$ ammonia acetate and $0.5 \%$ formic acid. The binary gradient started with $95 \% \mathrm{~B}$ decreasing to $80 \% \mathrm{~B}$ in $15 \mathrm{~min}$ where it was held for $20 \mathrm{~min}$. Thereafter the column was re-equilibrated to $95 \% \mathrm{~B}$ for $10 \mathrm{~min}$. The total run time was $30 \mathrm{~min}$, at a flow rate of $250 \mu \mathrm{L} / \mathrm{min}$, with a column oven temperature of $50{ }^{\circ} \mathrm{C}$ and a tray temperature of $10{ }^{\circ} \mathrm{C}$. The injection volume was $3 \mu \mathrm{L}$.

A LTQ-Orbitrap hybrid linear ion trap-Orbitrap mass spectrometer (Thermo Fisher Scientific, Bremen, Germany) equipped with an electrospray ion source was used. The instrument was operated in preview mode for parallel MS/MS spectra in the linear ion trap, while running the Orbitrap in full scan mode at 100,000 resolution $(\mathrm{m} / \mathrm{z} 400)$ from $\mathrm{m} / \mathrm{z} 350$ to 1000 in positive ESI mode. Helium was used as damping gas. From the FT preview scan, the 10 most abundant ions were selected in data dependent acquisition (DDA), fragmented in the linear ion trap analyzer and detected at nominal mass resolution. The following parameters were used for positive and negative MS/MS experiments: Normalized collision energy was $35 \%$, the repeat count was 2 and the exclusion duration $15 \mathrm{~s}$. The activation $\mathrm{Q}$ was at 0.2 and the isolation width 1.2. The spray voltage was set to $5 \mathrm{kV}$, the tube lens offset was at $120 \mathrm{~V}$ and the capillary temperature $250{ }^{\circ} \mathrm{C}$. Data analysis was performed by Lipid Data Analyzer software as described previously [23]. Shorthand annotation of lipid species was done according to LipidMAPS shorthand nomenclature [27]. Since oxidized phospholipids are not covered in the aforementioned publication we amended the existing shorthand nomenclature by putting the number of additional oxygens in parenthesis at the end of the moiety they are attached with. The number behind the colon of the oxidized moiety in this case reflects not only $-\mathrm{C}=\mathrm{C}-$ double bonds but also ring equivalents and carbonyl groups.

\section{Supplementary Materials}

Supplementary materials can be found at http://www.mdpi.com/1422-0067/16/04/8351/s1. 


\section{Acknowledgments}

The research leading to these results has received funding from the European Community's Seventh Framework Programme (FP7/2007-2013) under grant agreement no. 202272.

\section{Author Contributions}

Pia Sala, Sandra Pötz and Martina Brunner developed the HILIC chromatography and the mass spectrometric method within their consecutive master theses. Martin Trötzmüller co-supervised all three master theses and provided valuable chromatographic input. Alexander Fauland performed the reversed phase comparison measurements. Alexander Triebl contributed by data analysis of oxidized phospholipids. Jürgen Hartler optimized Lipid Data Analyzer for processing of oxidized phospholipids. Ernst Lankmayr supervised the work of Pia Sala and Sandra Pötz. Harald C. Köfeler supervised the work of Martina Brunner and put the manuscript together.

\section{Conflicts of Interest}

The authors declare no conflict of interest.

\section{References}

1. Lushchak, V.I. Free radicals, reactive oxygen species, oxidative stress and its classification. Chem. Biol. Interact. 2014, 224, 164-175.

2. Fruhwirth, G.O.; Loidl, A.; Hermetter, A. Oxidized phospholipids: From molecular properties to disease. Biochim. Biophys. Acta Mol. Basis Dis. 2007, 1772, 718-736.

3. Esterbauer, H.; Gebicki, J.; Puhl, H.; Jurgens, G. The role of lipid-peroxidation and antioxidants in oxidative modification of 1dl. Free Radic. Biol. Med. 1992, 13, 341-390.

4. Bochkov, V.N.; Oskolkova, O.V.; Birukov, K.G.; Levonen, A.L.; Binder, C.J.; Stockl, J. Generation and biological activities of oxidized phospholipids. Antioxid. Redox Signal. 2010, 12, 1009-1059.

5. Leitinger, N. The Role of Phospholipid Oxidation Products in Inflammatory and Autoimmune Diseases; Springer: New York, NY, USA, 2008; Volume 49, pp. 325-350.

6. Brame, C.J.; Salomon, R.G.; Morrow, J.D.; Roberts, L.J. Identification of extremely reactive gamma-ketoaldehydes (isolevuglandins) as products of the isoprostane pathway and characterization of their lysyl protein adducts. J. Biol. Chem. 1999, 274, 13139-13146.

7. Kofeler, H.C.; Fauland, A.; Rechberger, G.N.; Trotzmuller, M. Mass spectrometry based lipidomics: An overview of technological platforms. Metabolites 2012, 2, 19-38.

8. Uchikata, T.; Matsubara, A.; Nishiumi, S.; Yoshida, M.; Fukusaki, E.; Bamba, T. Development of oxidized phosphatidylcholine isomer profiling method using supercritical fluid chromatography/ tandem mass spectrometry. J. Chromatogr. A 2012, 1250, 205-211.

9. Lee, J.Y.; Lim, S.; Park, S.; Moon, M.H. Characterization of oxidized phospholipids in oxidatively modified low density lipoproteins by nanoflow liquid chromatography-tandem mass spectrometry. J. Chromatogr. A 2013, 1288, 54-62. 
10. Gruber, F.; Bicker, W.; Oskolkova, O.V.; Tschachler, E.; Bochkov, V.N. A simplified procedure for semi-targeted lipidomic analysis of oxidized phosphatidylcholines induced by uva irradiation. J. Lipid Res. 2012, 53, 1232-1242.

11. Watson, A.D.; Leitinger, N.; Navab, M.; Faull, K.F.; Horkko, S.; Witztum, J.L.; Palinski, W.; Schwenke, D.; Salomon, R.G.; Sha, W.; et al. Structural identification by mass spectrometry of oxidized phospholipids in minimally oxidized low density lipoprotein that induce monocyte/endothelial interactions and evidence for their presence in vivo. J. Biol. Chem. 1997, 272, 13597-13607.

12. Watson, A.D.; Subbanagounder, G.; Welsbie, D.S.; Faull, K.F.; Navab, M.; Jung, M.E.; Fogelman, A.M.; Berliner, J.A. Structural identification of a novel pro-inflammatory epoxyisoprostane phospholipid in mildly oxidized low density lipoprotein. J. Biol. Chem. 1999, 274, 24787-24798.

13. Samhan-Arias, A.K.; Ji, J.; Demidova, O.M.; Sparvero, L.J.; Feng, W.; Tyurin, V.; Tyurina, Y.Y.; Epperly, M.W.; Shvedova, A.A.; Greenberger, J.S.; et al. Oxidized phospholipids as biomarkers of tissue and cell damage with a focus on cardiolipin. Biochim. Biophys. Acta Biomembr. 2012, 1818, 2413-2423.

14. Liebisch, G.; Scherer, M. Quantification of bioactive sphingo- and glycerophospholipid species by electrospray ionization tandem mass spectrometry in blood. J. Chromatogr. B 2012, 883, 141-146.

15. Lisa, M.; Cifkova, E.; Holcapek, M. Lipidomic profiling of biological tissues using off-line two-dimensional high-performance liquid chromatography mass spectrometry. J. Chromatogr. A 2011, 1218, 5146-5156.

16. Schwalbe-Herrmann, M.; Willmann, J.; Leibfritz, D. Separation of phospholipid classes by hydrophilic interaction chromatography detected by electrospray ionization mass spectrometry. J. Chromatogr. A 2010, 1217, 5179-5183.

17. Reis, A.; Domingues, P.; Domingues, M.R.M. Structural motifs in primary oxidation products of palmitoyl-arachidonoyl-phosphatidylcholines by LC-MS/MS. J. Mass. Spectrom. 2013, 48, 1207-1216.

18. Stubiger, G.; Belgacem, O.; Rehulka, P.; Bicker, W.; Binder, B.R.; Bochkov, V. Analysis of oxidized phospholipids by maldi mass spectrometry using 6-aza-2-thiothymine together with matrix additives and disposable target surfaces. Anal. Chem. 2010, 82, 5502-5510.

19. Jonasdottir, H.S.; Nicolardi, S.; Jonker, W.; Derks, R.; Palmblad, M.; Ioan-Facsinay, A.; Toes, R.; van der Burgt, Y.E.M.; Deelder, A.M.; Mayboroda, O.A.; et al. Detection and structural elucidation of esterified oxylipids in human synovial fluid by electrospray ionization-fourier transform ion-cyclotron mass spectrometry and liquid chromatography-ion trap-ms3: Detection of esterified hydroxylated docosapentaenoic acid containing phospholipids. Anal. Chem. 2013, 85, 6003-6010.

20. Milic, I.; Hoffmann, R.; Fedorova, M. Simultaneous detection of low and high molecular weight carbonylated compounds derived from lipid peroxidation by electrospray ionization-tandem mass spectrometry. Anal. Chem. 2013, 85, 156-162. 
21. Davis, B.; Koster, G.; Douet, L.J.; Scigelova, M.; Woffendin, G.; Ward, J.M.; Smith, A.; Humphries, J.; Burnand, K.G.; Macphee, C.H.; et al. Electrospray ionization mass spectrometry identifies substrates and products of lipoprotein-associated phospholipase $\mathrm{A}_{2}$ in oxidized human low density lipoprotein. J. Biol. Chem. 2008, 283, 6428-6437.

22. Ishida, M.; Yamazaki, T.; Houjou, T.; Imagawa, M.; Harada, A.; Inoue, K.; Taguchi, R. High-resolution analysis by nano-electrospray ionization fourier transform ion cyclotron resonance mass spectrometry for the identification of molecular species of phospholipids and their oxidized metabolites. Rapid Commun. Mass Spectrom. 2004, 18, 2486-2494.

23. Hartler, J.; Trotzmuller, M.; Chitraju, C.; Spener, F.; Kofeler, H.C.; Thallinger, G.G. Lipid data analyzer: Unattended identification and quantitation of lipids in LC-MS data. Bioinformatics 2011, 27, 572-577.

24. Gross, M.L. Charge-Remote fragmentations-Method, mechanism and applications. Int. J. Mass Spectrom. Ion Process. 1992, 118, 137-165.

25. Pittenauer, E.; Allmaier, G. The renaissance of high-energy cid for structural elucidation of complex lipids: MALDI-TOF/RTOF-MS of alkali cationized triacylglycerols. J. Am. Soc. Mass Spectrom. 2009, 20, 1037-1047.

26. Harrison, K.A.; Davies, S.S.; Marathe, G.K.; McIntyre, T.; Prescott, S.; Reddy, K.M.; Falck, J.R.; Murphy, R.C. Analysis of oxidized glycerophosphocholine lipids using electrospray ionization mass spectrometry and microderivatization techniques. J. Mass Spectrom. 2000, 35, 224-236.

27. Liebisch, G.; Vizcaino, J.A.; Kofeler, H.; Trotzmuller, M.; Griffiths, W.J.; Schmitz, G.; Spener, F.; Wakelam, M.J.O. Shorthand notation for lipid structures derived from mass spectrometry. J. Lipid Res. 2013, 54, 1523-1530.

28. Fauland, A.; Kofeler, H.; Trotzmuller, M.; Knopf, A.; Hartler, J.; Eberl, A.; Chitraju, C.; Lankmayr, E.; Spener, F. A comprehensive method for lipid profiling by liquid chromatography-ion cyclotron resonance mass spectrometry. J. Lipid Res. 2011, 52, 2314-2322.

29. Folch, J.; Lees, M.; Stanley, G.H.S. A simple method for the isolation and purification of total lipides from animal tissues. J. Biol. Chem. 1957, 226, 497-509.

30. Ho, Y.L.; Chiu, J.H.; Wu, C.Y.; Liu, M.Y. Separation and determination of in vitro oxidized phospholipids by capillary zone electrophoresis. Anal. Biochem. 2007, 367, 210-218.

(C) 2015 by the authors; licensee MDPI, Basel, Switzerland. This article is an open access article distributed under the terms and conditions of the Creative Commons Attribution license (http://creativecommons.org/licenses/by/4.0/). 